\title{
"SCOUTISMO MIRANDO AL SUR": EL ORIGEN DEL MOVIMIENTO SCOUT A ESCALA COMPARADA. ARGENTINA Y BRASIL EN LAS PRIMERAS DÉCADAS DEL SIGLO XX
}

\author{
Maria Augusta Martiarena de Oliveira ${ }^{1}$ \\ Laura Marcela Méndez ${ }^{2}$
}

\section{RESUMEN}

Desde su origen en la primera década del siglo XX hasta los tiempos actuales, el scoutismo ha sido y es el mayor movimiento de infantes y jóvenes asociado a las actividades de tiempo libre del mundo. Aproximadamente setenta millones de muchachos y hombres y cuarenta millones de niñas y mujeres han sido o son miembros de los dos movimientos fundados por Baden Powell, los scouts y las guías. Actualmente cerca de nueve millones de boy scouts activos y seis millones de niñas y jóvenes se instruyen en la filosofía scout y sus prácticas en alrededor de cien países. El propósito central de esta ponencia es historiar -desde una perspectiva comparada- el surgimiento del scoutismo en dos países latinoamericanos, Brasil y Argentina, teniendo en cuenta cómo un movimiento internacional es resignificado en los Estados nacionales para construir nacionalidad y formar ciudadanos. La ponencia se dedica, en especial, al análisis de la utilización del scoutismo en las regiones sur de ambos los países. La perspectiva de la historia comparada que se propone intenta, -como ya lo había propuesto Marc Bloch a comienzos de los años $1960^{3}$-, analizar con procedimientos metodológicos específicos la unidad de un problema en dos sociedades, en este caso en la argentina y la brasilera, sin establecer un orden jerárquico o modélico de una sobre otra. Es pretensión de esta práctica historiográfica liberarse del eurocentrismo, las genarlizaciones y la naturalización del Estado-nación como espacio esencial de la historia, en la intención de aportar explicaciones causales, identificando singularidades y pautas comunes en los procesos históricos. El horizonte temporal seleccionado obedece a que, si bien el inicio del "Estado" argentino es previo, es recién a fines del siglo XIX cuando se profundiza su proceso de consolidación, periodo que coincide con la república brasilera y el surgimiento del scoutismo, concebido como una escuela moral de la infancia y la temprana juventud al servicio de ciertos valores morales y éticos. El análisis de coincidencias y divergencias entre ambos movimientos se hará atento a cuáles fueron sus principios rectores, quiénes fueron sus organizadores, cuáles sus primeras acciones y qué lugar tuvieron dentro de él la masonería en el caso de Brasil y la Liga Patriótica en la versión argentina. Libros scouts, actas fundacionales, fotografías, correspondencia y prensa nacional y regional serán los andamiajes que sostienen este recorrido, sustentado en los estudios culturales y en la idea de un Estado regionalizado, en el que la variable espacial condiciona itinerarios y procederes y, en el que en ambos sures (brasilero y argentino), el scoutismo fue valorado positivamente, asociado al "orden y progreso" y visibilizados en la esfera pública con carácter modélico. De esa forma, se percibe las utilizaciones políticas de las prácticas del scoutismo en regiones lejanas al centro político de los países estudiados, en el que el Estado ha utilizado instituciones de formación moral para la infancia y la juventud, como una manera de mantener la unidad nacional y fortalecer el Estado nacional, que en aquel momento se consolidaba, en el caso de Argentina, o un sistema político que se establecía, en el caso brasilero.

Palabras claves: scoutismo- Argentina-Brasil- historia comparada- 


\section{Los comienzos}

El teniente general Sir Robert Sthephenson Smyth Baden Powell , nacido en Londres en 1857, fue asignado -cuarenta y dos años más tarde- a defender la ciudad de Mafeking (Sudáfrica) de un prolongado sitio organizado por los Boer enemigos, oportunidad en la que utilizó los servicios de los jóvenes del grupo de mensajeros de la ciudad. Este hecho fue el punto de inspiración para la posterior creación del movimiento scout, al advertir el grado de eficacia y confiabilidad de estos jóvenes cuando eran entrenados y contenidos en ambientes de participación, respeto y obligación mutua.

Fue en 1907, después de consultas y entrevistas a pedagogos y a dirigentes de asociaciones juveniles, cuando Baden Powell organizó el primer campamento para enseñar a los chicos el arte de explorar los bosques y la disciplina del acecho. La denominación scout era utilizada en el ejército inglés para denominar a los soldados de primera línea que hacían exploraciones y tareas de espionaje preliminares en el territorio enemigo. Para diferenciarlos de éstos, se le antepuso la palabra "boys" y así también determinar el rango etario de los miembros de la agrupación. De esta manera se daba comienzo al movimiento mundial de los boy scouts.

El éxito fue tal que en 1908 se publicó en seis entregas quincenales "Escultismo para Muchachos", publicación que difundía la filosofía scout. A finalizar ese año, ya había sido traducida a cinco idiomas. Se trató de un movimiento jerárquico organizado a partir de los mandatos establecidos por su fundador, sumamente prescriptivo -pauta conductas deseables en diferentes escenarios y momentos de la vida-, con un fuerte vínculo con el entorno natural asociado al cuidado y la supervivencia y con un componente altamente moralizante, basado en el servicio al prójimo. Apeló a la fantasía, a la aventura y a la auto superación, rescatando el valor de todas las personas, que más allá de sus características individuales, encontrarían en la práctica del scoutismo la forma de convertirse en mejores sujetos, útiles para la sociedad.

Grupos scouts proliferaron en Inglaterra y casi simultáneamente, en decenas de países. Brasil y Argentina fueron unos de ellos.

\section{EI Scoutismo en Argentina y la Patagonia como tierra prometida}

Cuando en abril de 1909 Baden Powell visitó Argentina para dar una conferencia sobre la creación del movimiento fue recibido en el puerto de Buenos Aires por patrullas ya conformadas de manera informal un año atrás. Durante su estadía en el país planteó las bases del Scoutismo, aclarando que si bien en general se lo relacionaba con acciones militares, su interés estaba puesto en forjar hombres viriles y de carácter, que respondiesen a las necesidades de un nuevo ciudadano para el mundo. Confianza en sí mismo, autodisciplina, caballerosidad, lealtad y patriotismo eran algunas de las virtudes necesarias a desarrollar en los jóvenes para mitigar la holgazanería y el vandalismo. El contacto con la naturaleza y el profundo conocimiento de la misma, eran aspectos que permitían el desarrollo de las más altas cualidades humanas, tanto morales como físicas.

La propuesta de Baden Pawell fue muy bien acogida por la prensa, los sectores dirigentes y la opinión pública porteña. El movimiento scout argentino estaba en marcha. 


\section{La Asociación de Boy Scouts Argentinos}

La Argentina del centenario era un país fragmentado socialmente y escenario de movimientos sociales y políticos emergentes. La crisis política, económica y social a nivel mundial evidenciada tras la finalización de la primera guerra mundial fue el catalizador que impulsó a un grupo heterogéneo de personas a posiciones contrarrevolucionarias (Mc Gee, 2003).

Las movilizaciones de trabajadores acaecidas entre 1909 y 1910 y, especialmente, las de 1919-1921, hicieron que muchos argentinos creyeran que una revolución al estilo de la bolchevique era inminente en el país. Sin embargo, las organizaciones que surgieron tras esta amenaza no fueron meramente coyunturales, sino que se vinculaban con las tensiones asociadas con las políticas económicas implementadas en la segunda mitad del siglo XIX. Si bien hacia 1915, la Argentina era el ejemplo más exitoso del modelo económico liberal en América Latina, algunas de las consecuencias de ese crecimiento trastocaron la estructura social tradicional. Entre ellas, el surgimiento de un numeroso grupo migratorio asentado en las ciudades, el desequilibrio demográfico, el desarrollo del anarquismo, el socialismo, el movimiento obrero y el feminismo.

Los contrarrevolucionarios intentaron promover la estabilidad y su visión de la sociedad a través del fortalecimiento de la familia, la moral, la religión, la autoridad, la propiedad y el nacionalismo. Su ideario político se plasmó en la Liga Patriótica Argentina, conformada a comienzos de 1919.

La Liga manifestaba en sus propósitos que no respondería a intereses partidarios, sino que se abocaría a defender a la nación. Estimularía el sentimiento de argentinidad entre los habitantes del país, con independencia de su religión, su pertenencia política, su edad y su nivel de ingreso. Lucharía por más escuelas y mejores maestros, que enseñarían a los niños a amar y a respetar a la Argentina. Alentaría la celebración de las fiestas nacionales, promovería el respeto por las fuerzas armadas y concientizaría a la población del honor que significaba servir a las instituciones que protegían el hogar, el orden y la libertad. Según manifestara el presidente de la Liga, Manuel Carlés, quien ejerció ese cargo desde 1919 hasta su fallecimiento en 1946, en 1922 funcionaban 1400 brigadas en todo el país, 298 de ellas en territorio patagónico.

En este clima de ideas, la salvaguarda de la Patria tanto desde la moralidad como de la defensa contra sus enemigos adquirió prioridad. De ahí que estos grupos de nacionalistas apoyaran con ímpetu a instituciones vinculadas a la promoción de una particular cultura física que formara en estos principios. El scoutismo fue una de las asociaciones. La Patagonia, la tierra prometida.

El crecimiento del scoutismo en el país fue exponencial: de seiscientos scouts en 1915, el número ascendió a 20.000 en 1929 y alrededor de 40.000 diez años más tarde. Las compañías se extendieron desde Ushuaia hasta Jujuy, desde el Atlántico hasta la Cordillera de los Andes.

\section{Patagonia, entre la leyenda y el futuro}

En las tres primeras décadas del siglo XX puede observarse en las jóvenes comunidades patagónicas una transformación progresiva de la sociabilidad, a partir del surgimiento de asociaciones voluntarias. El aliento a la formación de grupos scouts formó parte del variado abanico de herramientas que la dirigencia nacional esgrimió en pos de la consolidación de la soberanía territorial concebida como sostén ineludible del Revista HISTEDBR On-line, Campinas, $n^{\circ}$ 63, p. 3-13, jun2015 - ISSN: 1676-2584 
status quo. Se priorizó la formación integral de la niñez y de la juventud, a través de la conquista del espacio público -escenario de un conjunto de conmemoraciones y rituales patrios- y de instituciones a las que el Estado propició y tuteló, convencido de su poder regenerador del ser argentino.

El scoutismo tuvo gran importancia para las poblaciones del sur argentino, tanto por las expectativas que la agencia estatal puso en ellas como medios de encauzamiento de una población signada por la extranjeridad o por el estigma del origen en el caso de los indígenas, como por el lugar que estas instituciones ocuparon al interior de las comunidades patagónicas como espacios de socialización, de ejercicio del poder, de institucionalización del espacio público y de construcción de un ideal de niñez y de juventud que se expresaría en la cultura física, signada por principios morales y valoraciones axiológicas, sobre los que se proyectaba el horizonte de expectativa futuro.

Los integrantes del movimiento scout fueron infantes y jóvenes escolarizados, habitantes de los centros urbanos y, por lo general, pertenecientes a sectores medios y medios bajos.

En los scouts fue contundente la presencia del ejército y las fuerzas del orden lo que, a nuestro juicio, constituye una posible llave de comprensión de los procesos de subjetivización de los territorios patagónicos y las lógicas de la praxis educativa, en un período en el que los gobernadores de origen militar fueron amplia mayoría en los Territorios Nacionales Patagónicos (Gentile, 2003).

Las comisarías locales fueron en muchos casos sedes de agrupaciones scouts y escenarios improvisados de stands de tiro; los dirigentes e instructores tanto del scoutismo como de los polígonos fueron la mayoría de las veces comisarios, subcomisarios o jefes militares.

A la asociación entre actividad física, salud y fortaleza de carácter se le sumaba un cuarto elemento: la percepción del potencial educador de la naturaleza. El lema "Amar la patria es conocerla" fue mucho más que un slogan de época: reflejaba la opinión mayoritaria de la prensa territoriana y la dirigencia acerca de que la naturaleza era fuente de inspiración de sentimientos nacionalistas.

El espacio geográfico y el paisaje se concebían como constructores de nacionalidad -por lo que promovían su estudio y conocimiento- y el cumplimiento de ciertos preceptos morales como base de la grandeza de la patria.

Los campamentos fueron concebidos como motores de la transformación educativa y como escuelas prácticas donde el niño aprendería a bastarse por sí mismo. El discurso médico sirvió como soporte para fomentar las actividades en el entorno natural, haciendo patente la percepción acerca de los peligros de vida urbana en relación a la formación integral de niños y jóvenes.

Se propiciaron intercambios y contactos con asociaciones de otras latitudes. Fue moneda corriente la recepción de grupos scouts en la cordillera neuquina y rionegrina durante la temporada estival. Estos viajes tenían como característico que no sólo se trataba de entablar relación con otras agrupaciones, sino conocer la historia, la geografía y la vida cultural de los lugares que visitaban, siendo frecuente que los cronogramas incluyeran recorridas de museos y sitios de interés histórico y cultural.

Sin lugar a duda, estas redes relacionales permitieron a los habitantes de la Patagonia en primer lugar sentirse más integrados a una comunidad nacional que trascendía los límites de su región -en un momento histórico y social en el que las distancias reales y simbólicas se magnificaban- y, por otro lado, afianzar rasgos identitarios propios, que permitían consolidad y constituir sesgos regionales y locales en 
un momento de gran avance de discurso que propendía a la instalación de un ser nacional homogéneo.

Las comunidades locales y la prensa también apoyaron a los scouts, participaron de sus cooperadoras y difundieron todas sus acciones. Grandes festejos se organizaban al arribo de contingentes scouts de vista en la región y siempre el aplauso y las banda de música locales acompañaba los desfiles de los batallones, concebidos como garantes de la defensa de la Patria ante un conflicto bélico, en el espacio público.

\section{EI Scoutismo en Brasil y en la región sur}

En Brasil, el scoutismo comenzó en el año de 1910, cuando se fundó el Centro de Boys Scout de Brasil, en Rio de Janeiro. Este movimiento había comenzado con los oficiales de la Marina, que se impresionaron con el nuevo método de educación complementaria en una visita a Inglaterra (Thomé, 2011). Después de la Primera Guerra Mundial, Brasil vivió una fase de nacionalismo muy fuerte. Algunos movimientos y campañas nacionalistas se iniciaron, con la finalidad de promover la elevación moral y política del país (Soauza, 2000). Fue en la asociación entre la educación cívica y el nacionalismo que ocurrió el entusiasmo por el scoutismo y su implantación. Además de los espacios educacionales, el scoutismo estaba entre los ideales preconizados por la Liga de Defensa Nacional.

La creación de los grupos de Boy Scouts en el sur de Brasil está relacionada a la campaña nacionalista del poeta Olavo Bilac. En las ciudades de Pelotas y Rio Grande, es posible decir que el espacio que el tema del scoutismo encontró en la prensa entre las décadas de 1910 y 1920 y la relevancia que ese tema logró en la sociedad, se debe a la campaña cívica de Bilac. En el año 1916, el poeta había estado en la ciudad de Pelotas y su visita había rendido muchos frutos para el movimiento nacionalista y republicano. En 1918, el Almanach de Pelotas dedicó una noticia a los orígenes del scoutismo en la ciudad. Con bases en los periódicos investigados, es posible afirmar que Fernando Osório ${ }^{4}$ fue la persona responsable de la instalación del primer grupo de Boys Scout, lo que ocurrió en 5 de noviembre de 1916 y fue uno de los resultados de la visita de Bilac. En el día de la creación del grupo de scoutismo, ocurrió un acto público:

$\mathrm{O}$ acto foi publico e festivo. No "stand" da sociedade do Tiro 31, perante avultada massa de povo, realizava-se a festa das Arvores. Olavo Bilac, que em propaganda cívica se achava nesta cidade, estava presente á brilhante cerimonia e enterrara, elle próprio, a primeira das arvores que naquelle dia seriam plantadas no campo do "Stand". Sessenta e seis jovens inscriptos aguardavam ali a creação do núcleo de escoteiro a que iam pertencer. Foi ainda o insigne poeta Olavo Bilac que proclamou realizada a ideia de Fernando Osório, e, tomando da espada deste, armou o primeiro escoteiro, proferindo a formula feliz que improvizara: "Armo-te Escoteiro do Brasil para a Honra, para a Virtude, para a Justiça e para a Bondade", (ALMANACH de Pelotas, 1918).

Además de esa noticia, el periódico "Opinião Pública" también se preocupó en difundir la historia de la instalación del scoutismo en la ciudad de Pelotas. El periódico deja claro la relación de la introducción del scoutismo por intermedio del "Tiro Brazileiro" (también conocido como "Tiro 31"): 
Creação de Escoteiros em Pelotas - O conselho diretor do tiro Brazileiro de Pelotas vae crear, annexa á esta associação, uma secção de escoteiros por iniciativa de seu instructor tenente Waldemar Schneider. Destina-a á educação physica e civica dos meninos de 11 a 16 annos, sem distinção de classe, attendendo a que o regulamento do Tiro Federal só admitte sócios a começar da idade de 16 annos, (OPINIÃO Pública, 10 de janeiro de 1916).

Además de la importancia de la creación de un grupo de scoutismo en la ciudad de Pelotas, el Almanach afirmó que la fundación en la ciudad fue pionera en relación a todo el estado del Rio Grande do Sul:

Organizado sob o regimen escolar, anexo á sociedade do Tiro 31, foi o primeiro grupo de escoteiros constituído neste Estado. Foi portanto Fernando Osorio o implantador do escotismo no Rio Grande do Sul, relevantíssimo serviço que lhe valerá a gratidão de seus conteporaneos (sic) e das gerações beneficiadas com as vantagens da utilíssima insitutição de Baden Powell, (ALMANACH de Pelotas, 1918, p.185)

Las afirmaciones del Almanach de Pelotas demuestran, una relación del scoutismo con la organización escolar. Además de eso, evidencia la relación de este con la formación moral, cívica y física de los jóvenes y sus semejanzas con los objetivos de la disciplina de Educación Física. El scoutismo se integraba perfectamente al contexto educativo de las primeras décadas del siglo XX, en que se preconizaba una formación integral del individuo. De acuerdo con Nascimento (2004), el suceso del scoutismo, tanto en ámbito nacional como mundial, estaba relacionado a un contexto de crisis y de valorización de la idea de nación. Además, se desarrollaron nuevas prácticas pedagógicas que tenían por objetivo formar a los estudiantes moralmente. El scoutismo constituyese, aún, como herramienta para disciplinar a los cuerpos. Para Scharagrodsky (2001), las marchas y evoluciones militares tendieron, además de a la uniformidad, al orden y a la disciplina del cuerpo infantil. Además, el autor afirma que fueron importantes para configurar un determinado orden corporal masculino que se asociaba a la hombría, al valor, al vigor, a la fuerza, al coraje y a la formación del carácter. Tales ideas se encuentran relacionadas, también, con el movimiento higienista. Con que, la ideas pedagógicas trajeron nuevas preocupaciones que relacionaron el ambiente escolar con la higiene y el saneamiento. Según Stephanou (2005), en las primeras décadas del siglo XX, se le dio mucho crédito a la educación y su función de saneamiento del medio y del hombre, con la idea generalizada de que muchas enfermedades eran causadas por la ausencia de "la investigación racional y la educación " de la gente. Sin embargo, la autora afirma que la solución no era ningún tipo de educación, sino una educación "completa" la educación "integral" y se basa en el conocimiento científico podría dar cuenta de tal empresa.

El contexto global permitió a la formación de una ideología educativa que tenía el objetivo de educar la mente y el cuerpo. Esos ideales educativos eran convenientes a la situación política que tanto Brasil como Río Grande do Sul vivían. Se puede ver que, tanto a nivel nacional como regional, la clase gobernante dirigió sus esfuerzos para la formación del "ciudadano" en busca de lograr la uniformidad, mientras que eran cada vez más evidentes las diferencias sociales derivadas del Imperio y agravadas en la República. Existía, en particular, la tendencia a "urbanizar" y "desinfectar" las personas, 
lugares destinados a ciertos grupos y la realización de una selección/exclusión de los habitantes. De esa manera:

Uma educação física, intelectual e moral da mocidade, com a finalidade de possibilitar ao espírito todas as noções necessárias para melhor garantir a ordem, se colocou como central para que todos os homens tivessem consciência de seu papel social. Em outras palavras, a educação foi vinculada à formação do cidadão", (Corsetti, 1997, p.167).

\section{Rio Grande do Sul: la distancia del centro político brasileño}

Es posible decir que, históricamente, la región sur tardó en integrarse al territorio brasileño. En el período colonial, la región, por el Tratado de Tordesillas, pertenecía a la corona española y no a la portuguesa. Los territorios se fueron agregando a los de la corona portuguesa, a través de la Unión de las Coronas Ibéricas, en el gobierno de Felipe II y, más tarde, por los tratados diplomáticos que entregaban la región de las Reducciones Jesuíticas de Brasil a los portugueses y Colonia del Sacramento a los Españoles (Tratados de Madrí y Santo Ildefonso, ambos del siglo XVIII). Después de la independencia de Brasil, en el siglo XIX, Rio Grande do Sul fue lugar de la Revolución Farroupilha, en que se declaró independiente la "República Rio-Grandense". Además de todo, algunos estudiosos entienden que la identidad de la región sur, al mismo tiempo que influenciada por el centro político de Brasil, también es marcada por la fuerte intervención de los vecinos del Río de la Plata.

En fines del siglo XIX, cuando Brasil se convirtió en una república, fue considerado importante formar los ciudadanos para el nuevo régimen. La región sur ya poseía las características anteriormente mencionadas y, agregado a eso, la cuestión de la inmigración era todavía muy fuerte y muchas regiones de Rio Grande do Sul, los inmigrantes mantenían su idioma de origen. A esa situación, se entiende, entonces, que es necesario difundir los ideales nacionalistas, así como el idioma nacional. Una de las estrategias de divulgación del ideario republicano y patriótico fue formar a los niños y a los jóvenes, a través de la escuela. En ese sentido, el Scoutismo se estableció en la región sur, después de la campaña cívica de Olavo Bilac, como una formación complementaria a la escuela.

Además de todo, los rasgos del Scoutismo eran muy adecuados para que los jóvenes se convirtieran en ciudadanos obedientes al poder central. La cuestión de la militarización de los jóvenes es muy importante y puede ser percibida no sólo por el uniforme utilizado por los boy scouts, por su postura. El deseo de la militarización es más que un discurso, y no está solamente entre las líneas, pero era parte de una ideología oficial, que se incrementó con el contexto de la Primera Guerra Mundial, en que fueron instalados los scouts en la ciudad de Pelotas y la realización de la campaña cívica de Olavo Bilac. El Scoutismo representa la preocupación con la seguridad nacional:

O movimento geral que se manifesta no paiz a favor da defesa nacional, reorganizando-se sociedades de tiro, augmentado, de uma maneira digna de encomicos, os voluntários de manobras, faz nascer a esperança de, em futuro próximo, ser o Brasil uma nação regularmente forte, formada d'homens capazes de defender o solo pátrio com ardor e intelligentemente, (OPINIÃO Pública, 31 de agosto de 1916). 
Además del scoutismo, el contexto global de la guerra condujo al resurgimiento de las sociedades de tiro, que tenía como objetivo transformar a Brasil en un país preparado para protegerse en cualquier conflicto armado. La militarización de los niños y el país no se percibe como un problema, sino como una forma de defensa: "Esse movimento não trará em resultado a militarisação do paiz com o fim de aggredir, mas sim a generalização dos conhecimentos de guerra indispensáveis a um povo que quer subsistir, mantendo intacta a sua honra e integral o seu território", (OPINIÃO Pública, 31 de agosto de 1916). Además, el scoutismo era visto como um médio de preparar los niños para la ciudadanía y para la defensa del país: "Mas é preciso ir mais longe, é necessário preparar a creança para que com mais facilidade possa, mais tarde, usufruir as grandes vantagens das linhas de tiro", (OPINIÃO Pública, 31 de agosto de 1916). Esto no se produjo solamente en el sur del país, como es posible percibir abajo:

\begin{abstract}
A militarização da infância ressurgia de forma mais sistematizada e racionalizada sob os auspícios dos órgãos da administração do ensino público e a Associação Brasileira de Escotismo. As práticas cívicomilitares em voga nas escolas primárias atendiam, assim, a múltiplos propósitos: fosse a perpetuação da memória histórica nacional, a exibição das virtudes morais e cívicas inscritas na obra formativa escolar, a ação educadora da escola para o conjunto da sociedade ou a expressão do imaginário sociopolítico da República, (SOUZA, 2000, p.116).
\end{abstract}

La idea de la institución del scoutismo se hizo más intensa, de modo que, en la ciudad de Pelotas, no encontró opositores ni siquiera en el periódico "Rebate", que tan fuertemente se opuso a los gobiernos municipales y provinciales. Acerca de los Boy Scouts, sólo se ha reportado su participación en las celebraciones cívicas de la Fiesta de la Bandera, en el que "fizeram exercícios na praça da Republica, de hygiene, telegraphia e tratamento de ferimentos", (REBATE, 20 de noviembre de 1916). Es posible acreditar también que el desarrollo del Movimiento Scout en la ciudad de Pelotas se produjo de forma concomitante con otras partes de Brasil, incluyendo Sao Paulo. Esta provincia fue considerada ejemplar, por lo que la crítica con respecto a los batallones de infantes se hizo más efusivo a partir del momento en que había perdido su prestigio en este lugar, no durante la Primera República. Con respecto al scoutismo, por el contrario, esta se ha configurado en una novedad y fue ampliamente aceptado por los estudiosos, pues representaba el nacionalismo y la educación moral y cívica de los ciudadanos.

Además de formar a los niños y los jóvenes, las Escuelas de Boy Scouts llevaba sus conmemoraciones al espacio público. Así, su función de sanear a los cuerpos de los jóvenes se extendía para la diciplinarización de la población general, que accedía a las presentaciones de los Scouts.

\title{
Comentarios finales
}

El scoutismo como institución contó con el apoyo y beneplácito de la dirigencia argentina y brasilera de las primeras tres décadas del siglo XX, al concebirlo como un movimiento que cooperaría con la regeneración moral que exigían los países, sostendría el statu quo político y social y ayudaría a defenderse de los enemigos internos y externos.

Revista HISTEDBR On-line, Campinas, $n^{\circ}$ 63, p. 3-13, jun2015 - ISSN: 1676-2584 
El movimiento se constituyó en ambas naciones con el beneplácito de la Liga Patriótica Argentina y de la Liga de Defensa Nacional en Brasil en un contexto en el que la inminencia del primer conflicto bélico mundial exigía de niños y jóvenes su formación como soldados civiles, los conflictos internacionales y los fenómenos migratorios alertaban y generaban políticas nacionalistas y se aumentaba la posibilidad de acceso a la educación pública básica, que por ley incluía a más niños y niñas en los procesos de escolarización.

El movimiento scout se hizo fuerte en los extremos sur de ambos países. En la Patagonia argentina, el scoutismo fue de gran relevancia por su temprana aparición, su abultada cantidad de miembros y su contundente participación en el espacio público. Su desarrollo permite observar rituales y representaciones sociales vehiculizadas por los lazos asociativos que advierten cómo un reducido número de vecinos percibidos como los "notables" del espacio urbano tejieron redes políticas, sociales y económicas al compás de las prácticas corporales y los preceptos morales transmitidos en las prácticas scouts.

La geografía de la Patagonia fue a la vez motivo para estar alerta en su defensa, medio para generar patriotismo -a partir del conocimiento y admiración de las bellezas naturales- y un lugar para regenerar a niños y jóvenes en oposición a los peligros emergentes de los modos de vida urbanos. El contacto con asociaciones scouts de otras latitudes permitió construir sesgos de identidad local/regional a la vez que sentidos de pertenencia a la comunidad nacional.

El scoutismo fue apoyado por la Liga Patriótica Argentina, la prensa, los funcionarios del Estado, las instituciones educativas y la mayoría de la sociedad, en el convencimiento de que las prácticas corporales desplegadas cumplían la función de pedagogización social, al moralizar los niños, fortalecer sus cuerpos y reforzar su virilidad.

Así como en Patagonia, la introducción del scoutismo en el sur de Brasil fue ampliamente apoyada, tanto por las instituciones gubernamentales, como por la prensa y sociedades de Tiro. La distancia de Rio Grande do Sul hacia el poder central fue motivo de preocupación para la defensa nacional. Además de todo, la región ha recibido un número muy grande de inmigrantes. Como fue anteriormente mencionado, los grupos de Scouts fueron creados a partir de la campaña cívica de Olavo Bilac y ocurrieron contemporáneamente en el centro y en el sur del país.

En Brasil, la militarización de la infancia y de la juventud no fue comprendida como un problema, sino como una necesidad relacionada con la defensa nacional. De esa forma, los ideales patrióticos y nacionalistas encontraron en las Escuelas de Boy Scouts instituciones complementarias muy importantes que, además de disciplinar a los niños y jóvenes, disciplinaba a las persones que accedían las presentaciones de los scouts.

Pensar al scoutismo desde un enfoque regional y comparado, permite resquebrajar la idea del scoutismo como movimiento homogéneo y monolítico y concebirlo como un conjunto de agrupaciones que, más allá de historias principios y preceptos compartidos, constituyen realidades situadas y diferenciadas en relación a la etnia, la edad y el género de sus integrantes.

\section{REPOSITORIOS Y FUENTES:}


Conferencias del seminario Francisco P. Moreno de la Sociedad Científica Argentina. Año 1947. Buenos Aires, Talleres Gráficos de Administración General de Parques Nacionales y Turismo 1951.

Labor Parlamentaria del Perito Doctor Francisco P. Moreno. H. Senado de la Nación. Buenos Aires, Secretaría Parlamentaria. División Publicaciones. Buenos Aires, Imprenta del Congreso de la Nación, 1995.

Colección Francisco P. Moreno. Museo De La Patagonia "Francisco P. Moreno". San Carlos De Bariloche. (Citado como A. M.)

Colección Emilio Frey. Museo De La Patagonia "Francisco P. Moreno". San Carlos de Bariloche.

Periódico La Nueva Era. Carmen De Patagones. 1912-1945. Museo Histórico de la provincia de Río Negro Andrés Tello, Viedma, Río Negro.

Periódico El Territorio. 1933-1935. Neuquén. Sistema Provincial de Archivos del Neuquén.

Periódico Neuquén. 1928-1938. Neuquén. Sistema Provincial de Archivos del Neuquén.

Diario "Río Negro". 1912- 1945. Archivo Del Diario "Río Negro”, General Roca, Río Negro.

“Caras y Caretas. Seminario festivo, literario, artístico y de actualidades", 1898-1939. Buenos Aires.

ALMANACH de Pelotas, 1918. Archivo de la Biblioteca Pública Pelotense, Pelotas.

Diario Opinião Pública, 1896-1962, Archivo de la Biblioteca Pública Pelotense, Pelotas.

Diario O Rebate, 1914-1923, Archivo de la Biblioteca Pública Pelotense, Pelotas.

Bibliografía

GAYOL, SANDRA, MADERO, MARTA (2007) Formas de Historia Cultural, Buenos Aires, Prometeo.

AISENSTEIN, ANGELA, SCHARAGRODSKY, PABLO (2006) Tras las huellas de la Educación Física Escolar Argentina. Cuerpo, Género y Pedagogía. 1880-1950, Buenos Aires, Prometeo.

GARCÍA, NORMA (2001) "Pensar y hacer la política: los nacionalistas entre la idea y la organización, Neuquén en los '30", en PRISLEI, LETICIA (comp.) Pasiones Sureñas. Prensa, cultura y Política en la Frontera Norpatagónica (1884-1946), Buenos Aries, Prometeo, pp. 189-224.

MCGEE DEUSTCH, SANDRA (2005) Las derechas. La extrema derecha en la Argentina, el Brasil y Chile, 1890-1939, Buenos Aries, Universidad Nacional de Quilmes.

MÉNDEZ, LAURA, directora, (2011) Historias en movimiento. Cuerpo, educación y tiempo libre. Rosario, Prohistoria.

Revista HISTEDBR On-line, Campinas, $n^{\circ}$ 63, p. 3-13, jun2015 - ISSN: 1676-2584 
ROZENGARDT, RODOLFO Y ACOSTA FERNANDO (2005) Historia de la Educación Física y sus instituciones: continuidades y rupturas, Buenos Aires, 2011, pp.43-68.

CORSETTI, Berenice. O poder em migalhas - a escola no Rio Grande do Sul na Primeira República. Santa Maria, Universidade Federal de Santa Maria, 1997.

NASCIMENTO, Adalson de Oliveira. Educação e civismo: movimento escoteiro em Minas Gerais (1926-1930). Revista Brasileira de História da Educação, n 7, jan./jun., 2004.

SCHARAGRODSKY, p. Cuerpo, género y poder en la escuela. El caso de la Educación Física Escolar Argentina (1880-1930). Estudos Ibero-Americanos, Porto Alegre: PUCRS, v. XXVII, n. 2, p. 121-151, 2001.

SOUZA, Rosa Fátima de. A militarização da infância: Expressões do nacionalismo na cultura brasileira. Cadernos Cedes, ano XX, no 52, novembro/2000.

STEPHANOU, Maria. Discursos médicos e a educação sanitária na escola brasileira. In: STEPHANOU, Maria; BASTOS, Maria Helena Câmara (orgs.). Histórias e memórias da educação no Brasil, vol. III: século XX. - Petrópolis, RJ: Vozes, 2005, p.142-164.

THOMÉ, Nilson. Escotismo: história de uma prática educativa extra-escolar. In: Anais do VI Congresso Luso-Brasileiro de História da Educação, Uberlândia, 2006.

\footnotetext{
${ }^{1}$ Profesora Instituto Federal de Educação, Ciência e Tecnologia do Rio Grande do Sul/Campus Osório martiarena.augusta@gmail.com; augusta.martiarena@osorio.ifrs.edu.br

${ }^{2}$ Docente e investigadora de la Universidad Nacional del Comahue. Miembro del CEHIR-ISHIRCONICET, nodo Comahue. Centro Regional Universitario Bariloche, Argentina. lauramendezbari@gmail.com

${ }^{3}$ Marc Bloch, "Pour une Historie Comparée des socités européennes", en Melantes historiques, vol 1, S.E:V:P:E:N; París, 1963, pp. 16-40.

${ }^{4}$ Fernando Osório fue un estudioso pelotense, profesor de importantes instituciones educacionales de la ciudad de Pelotas, además de creador del Tiro 31, donde se originó la primera escuela de scoutismo de la ciudad.

${ }^{5}$ El Tiro de Guerra 31 fue fundado en la ciudad de Pelotas en 1903 y tenía como objetivo "culto cívico da integridade, da ordem e do progresso da Pátria [...]”, (OSÓRIO, 1962, p.171).
}

Recebido: $\quad$ setembro-15 Aprovado: setembro-15 